Review ARticle

\title{
GENERALITY AND UNIFORMITY IN A RULE-BASED PARSER
}

\author{
KENSUKE TAKONAI \\ Tokyo Metropolitan University*
}

Construal, by Lyn Frazier and Charles Clifton, Jr., The MIT Press, Cambridge, MA, 1996, x+230pp.

\section{Introduction}

For the past two decades Lyn Frazier and her colleagues have always been in the central position in the study of the human sentence processing mechanism, and have published a host of important and influential articles in which they propose and defend a number of structurallybased parsing principles, most notably, Minimal Attachment and Late Closure. In this book, however, Frazier and Clifton (henceforth F\&C) state that their purpose "is neither to defend the structural parsing principles nor to attack them. Rather, it is to go beyond them." (p. 2)

One thorny problem that pressed them to "go beyond" the structural parsing principles seems to be the apparent inapplicability of Late Closure in certain relative clause constructions in Spanish discovered by Cuetos and Mitchell (1988). Late Closure, as defined in (1), predicts that in sentences such as (2), the relative clause will be preferentially attached to the lower NP, del coronel, which is the phrase "currently being processed":

(1) Late Closure: If grammatically permissible, attach new items into the clause or phrase currently being processed (i.e., the clause or phrase postulated most recently).

(p. 9; Frazier (1987: 562))

* I am grateful to two anonymous $E L$ referees for comments and advice. 
(2) El periodista entrevisto a la hija del coronel que tuvo el accidente.

'The journalist interviewed the daughter of the colonel who had had the accident.'

(Cuetos and Mitchell (1988: 77))

However, it turned out that, contrary to this prediction, Spanish readers showed the opposite bias: they tended to interpret the higher NP, la hija, as the person who had the accident. This finding is challenging since Late Closure purports to be a universal parsing strategy which applies to any type of construction in any particular language.

Confronted with this and other recalcitrant problems, F\&C concede that Late Closure is not as general a principle as was supposed to be, and suggest that its limited applicability follows from a principled distinction between phrase types: although some types of phrases are parsed following Late Closure, certain other types of phrases are not, but instead subject to a different process. To make this idea explicit, they explore data from several constructions to which relatively little or no attention has hitherto been paid, and attempt to develop and confirm what they call the "Construal Hypothesis."

\section{The Construal Hypothesis}

\subsection{Basic Assumptions and Postulates}

It is generally assumed that, in the course of interpreting a sentence, we convert a string of words into something like a phrase marker and compute all the structural relationships within it. The human parser must then be able to construct a representation in which (immediate) dominance and sisterhood relations among phrases are fully specified. Usually, the construction of such a representation is considered to be performed without delay, i.e., as soon as the input items to be analyzed are encountered (cf. Frazier and Rayner's (1988:263) "Left-to-Right Constraint").

The essence of F\&C's Construal Hypothesis consists in admitting a certain restricted form of delay: it allows the parser to postpone certain structural decisions until sufficient information becomes available, and to initially build an indeterminate or underspecified representation in which certain dominance and sisterhood relations can be left unspecified. They write:

... certain phrases, instead of being attached into a fully determinate syntactic phrase marker using only structural information, 
are associated with a particular structural domain and interpreted using both structural and nonstructural information.

(p. 29; their italics)

In order to give an empirical content to the hypothesis, it is necessary to make explicit how the terms "certain phrases," "association," "domain," and "nonstructural information" are to be defined. Let us see how $\mathrm{F} \& \mathrm{C}$ do this.

First, they divide the phrases which serve as the input to the parser into two distinct types, i.e. primary and nonprimary (secondary) phrases:

(3) Primary phrases and relations include

a. the subject and main predicate of any $(+$ or -$)$ finite clause.

b. complements and obligatory constituents of primary phrases.

Primary phrases are attached into a phrase marker with their mother and sister nodes fully specified. They are parsed, as they are encountered, following general structural parsing principles (Minimal Attachment, Late Closure, and the Minimal Chain Principle), and receive a single immediate analysis even when they involve a temporary ambiguity. So, as far as primary phrases are concerned, the parser abides by the "First Analysis Constraint," i.e. adopts the first structural analysis available, typically the one that requires the postulation of the fewest nodes or permits quick structuring of new items (pp. 8-9; cf. Frazier (1987: 564-565), Frazier and Rayner (1988: 263)).

Nonprimary phrases, on the other hand, are parsed quite differently; they are immune to the operations of structural parsing principles and initially connected only loosely to a domain which can potentially contain more than one attachment site; they are exempt from the First Analysis Constraint but instead follow the "Construal Principle":

(4) Construal Principle

a. Associate a phrase XP that cannot be analyzed as instantiating a primary relation into the current thematic processing domain, and

b. Interpret XP within that domain using structural and non-structural (interpretive) principles.

(5) Current thematic processing domain

The current thematic processing domain is the extended maximal projection of the last theta assigner. (pp. 41-42, 
152; for extended projection, see Grimshaw (1993))

Nonprimary phrases correspond to optional adjunct phrases and include adverbs, adjunct PPs, relative clauses (both extraposed and nonextraposed), and adverbial clauses. Constructions involving them are expected to exhibit no parsing preferences as described by Minimal Attachment and Late Closure since the First Analysis Constraint applies only to primary phrases. The relative clause in (2) above, for example, is a nonprimary phrase and therefore, according to the Construal Hypothesis, need not be attached to the immediately preceding $\mathrm{NP}$, del coronel, as dictated by Late Closure; instead, it is initially associated into the larger NP (a) la hija del coronel, which is the extended maximal projection of (what $\mathrm{F} \& \mathrm{C}$ take to be) the last theta assigner hija, as in (6):

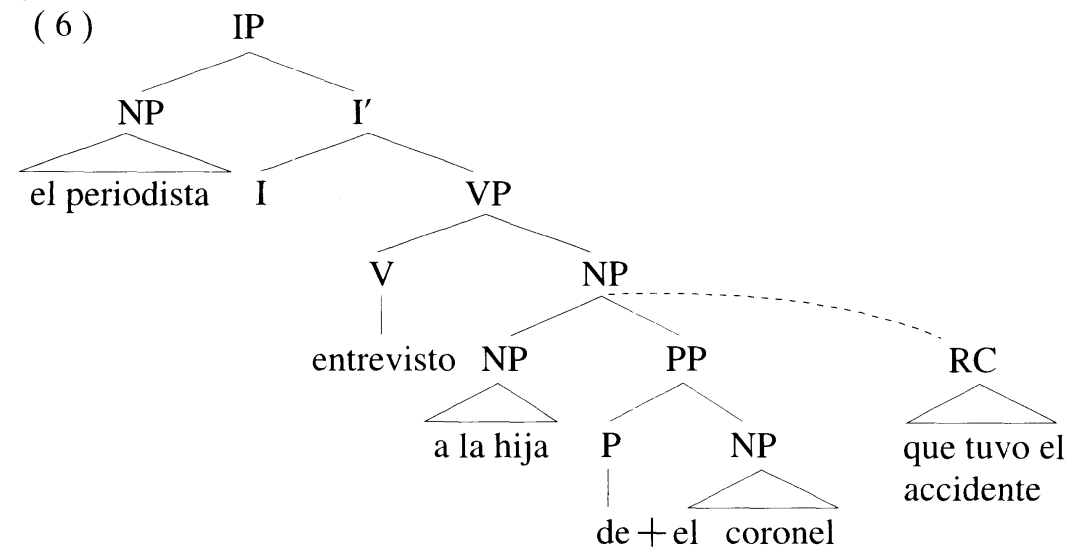

Association, which is indicated by a dashed line, is a syntactic operation that relates a nonprimary phrase to the larger structure without specifying which of the nodes within the current thematic processing domain is the immediate mother for the phrase. Put differently, association makes a nonprimary phrase a temporary orphan but places it in the custody of a fixed set of potential mothers, one of which is later chosen as the genuine mother. In the present example, the relative clause can potentially choose either of the NPs within the domain as its attachment site, its ultimate choice being determined by semanticpragmatic interpretive processes. Since no structurally preferred analysis is initially assigned to nonprimary phrases, no garden path is predicted to occur even when their final attachment analysis flouts structural parsing principles. Hence the Construal Hypothesis predicts 
that high attachment can be no less preferred than low attachment, depending on semantic or pragmatic factors, and accomodates apparent problematic data from Spanish without discarding Late Closure.

\subsection{Examples of Construal}

Let us see more closely how the Construal model works by examining a couple of examples from English. First, consider (7):

(7) Yesterday they gave me the sweater of cotton that was illegally imported.

Late Closure predicts that the relative clause that was illegally imported should more preferentially be attached to the lower of the two potential head NPs, cotton, than to the higher one, the sweater, and that the high attachment reading cannot be obtained without difficulty. The Construal Hypothesis, on the other hand, predicts that, being a nonprimary phrase, it will be initially associated into the entire IP, which is the extended maximal projection of the last theta assigner gave, that either the sweater or cotton can serve equally well as its host since they are in the same domain, and that no difficulty will be observed even when the higher NP is chosen as the head. The data from a questionnaire study suggest that the high attachment reading is in fact preferred, contrary to the prediction by Late Closure. Although the data do not contradict the Construal Hypothesis, it remains to be explained why higher attachment is preferred. $\mathrm{F} \& \mathrm{C}$ claim that the choice of the head for a relative clause is influenced by a variety of nonstructural factors including referentiality of the NPs, plausibility, discourse salience, and so on. In the present case, an interpretive principle, as formulated below, provides a source of the observed preference:

\section{( 8 ) Referentiality Principle}

The heads of some maximal projections are "referential" in the sense that they introduce entities (e.g., discourse participants) into the discourse model (at least temporarily) or correspond to already existing discourse entities. Restrictive modifiers (e.g., restrictive relative clauses) preferentially seek hosts that are referential in this sense. (p. 74)

In (7) the higher NP, the sweater, is referential whereas the lower NP, cotton, is not; the relative clause therefore chooses the former following (8), and the result is the apparent Early Closure reading.

Now consider the following sentence containing an extraposed relative clause: 
(9) The boy bought the shirt today that the girl liked so much. The Construal Hypothesis predicts that the extraposed relative clause will be associated into the extended maximal projection of bought, i.e. IP, and that, since it contains both the subject and the object, either NP should be available as the host for the relative clause. In this case, however, most perceivers show an overwhelming preference for the object-host interpretation. $\mathrm{F} \& \mathrm{C}$ conjecture that this will be accounted for by an interpretive principle which states that a relative clause is preferentially taken to modify a focused phrase, on the assumption that the object NP is more likely to receive focus than the subject NP, which is typically unfocused (p. 101; cf. Schafer et al.'s (1996) "Focus Attraction Hypothesis").

Construal also applies to adverbial clauses such as (10):

(10) John didn't leave because he was angry.

The because clause, which does not instantiate a primary relation, will be associated to the extended projection of leave, the last theta assigner. This association permits it to be ultimately attached to either the main IP or VP. Which node will be chosen is determined by nonstructural factors. F\&C (p. 66) propose the "Immediate Interpretation Principle" as one such factor operative in this case, according to which the main clause is interpreted immediately as it is processed, before the because clause is encountered: leave is interpreted under the scope of negation. This interpretation would have to be revised if the because clause were to be attached low to VP, as predicted by Late Closure. High attachment to IP, on the other hand, requires no revision of the initial interpretation. Hence, on the assumption that structures requiring the fewest revisions of the already assigned interpretation are favored (the Minimal Revisions Principle, p. 55), the because clause is predicted to be attached to IP. This is consistent with experimental data (end-of-sentence "got-it" responses).

These examples clearly demonstrate an important tenet of the Construal Hypothesis, namely that a variety of nonstructural factors may govern initial preferences in the interpretation of nonprimary phrases. It should, however, be emphasized that this hypothesis does not deny the existence of purely structural parsing principles; they are operative and they do influence initial preferences. It only claims that their domain of application becomes narrower: they apply selectively to primary phrases. 


\subsection{Summary}

As mentioned at the outset, $\mathrm{F} \& \mathrm{C}$ aimed to go beyond simple, structurally-based parsing principles such as Minimal Attachment and Late Closure. And what they found beyond them, the Construal Hypothesis, enabled a novel perspective on the human sentence processing mechanism. The language comprehension system that emerged incorporates a clear division of labor: one component of the processor is responsible only for primary phrases, analyzing them using structurally-based preference principles, while another component deals exclusively with nonprimary phrases and interprets them in terms of a number of nonstructural principles.

F\&C's hypothesis that different sorts of parsing decisions are made for different types of phrases is certainly interesting, and ought to be valued to the extent that it focuses on the formulation of semanticpragmatic interpretive principles that have received relatively little attention and explores new constructions containing adjunct phrases. From a different point of view, however, their hypothesis is undesirable in that it jeopardizes the generality and uniformity of the parsing mechanisms; the parser can no longer operate uniformly since it performs attachment analyses in some cases but executes a fundamentally different operation in others, and its decision principles are no longer fully general since they apply to only one class of phrases. Such a claim is weaker and less exciting than the one that maintains full generality and uniformity of the operations of the human parser. In the following section I will make some modifications to the Construal Hypothesis, and suggest an alternative that preserves the full generality and uniformity of the parser.

\section{Rule-Based Parsing without Construal}

\subsection{Relation between the Parser and the Grammar}

F\&C (p. 27) claim that their theory assumes a "principle-based" grammar, in particular, some version of Government-Binding (GB) theory. It is doubtful, however, whether their processing theory does justice to this claim. For one thing, it makes only restricted use of the notions and principles of GB theory: although it employs notions such as theta-assigners, heads, and maximal projections, no crucial reference is made to the Theta Criterion, the Projection Principle, Spec-head agreement, head-government, binary branching, Case filter, or Sub- 
jacency. Furthermore, despite their sporadic reference to the level of Logical Form (see pp. 48, 54, 162, 171, 204), F\&C never present any precise analysis to show the significance of its existence and derivation during on-line sentence comprehension. Accordingly, there seems to be no getting rid of the impression that $\mathrm{F} \& \mathrm{C}$ have a poor case for their principle-basedness claim.

But more importantly, F\&C's assumption of a principle-based grammar, when combined with their use of phrase structure rules, makes it impossible to attain a transparent relation between the grammar and the parser (for transparency, see Berwick and Weinberg (1984: Ch. 2) and Fodor (1989: 177-186)). F\&C (pp. 24-27) persist in using a set of phrase structure rules as the data structure of the parser; in fact, they must have recourse to rules, for their structural parsing principlesMinimal Attachment, Late Closure, and the Minimal Chain Principleare assumed to follow from the way the parser accesses the rules and chooses the first available analysis, i.e. from the parser obeying the "First Analysis Constraint." They write:

Since ... all three principles may be seen as consequences of a deeper principle, they need not be specifically stipulated. Rather, they follow from the claim that the parser constructs syntactic representations by applying rules (like phrase structure rules) that create a single enriched tree structure. If all rules are applied in parallel to incoming words (and to existing structure), and if the first way of relating each new word to the existing structure is chosen as the initial analysis, the initial analysis generally will be that which relates the new material to the most available old material using the smallest number of new nodes (or links in a chain; see Frazier and Fodor 1978).

(p. 9 ; footnote omitted)

It is obvious, therefore, that F\&C's theory (the "garden path model") is essentially founded on a "rule-based" grammar. The notions and principles of GB theory are, despite the alleged principle-basedness, never realized directly in the parsing process; to be accessible to the parser and applicable on-line, they must first be modified or "precompiled" into phrase structure rules (pp. 23-27; Frazier (1989)). The intervention of such a precompilation process renders the relation between the grammar and the parser indirect and opaque, which fact in turn makes it difficult to maintain that percievers interpret sentences efficiently on the basis of their knowledge of grammar. 
In order to attain transparency, then, it seems that $\mathrm{F} \& \mathrm{C}$ have to abandon either the principle-basedness assumption or the assumption of rule-based operation of the parser. Since F\&C's model does not make full use of the notions and principles of GB theory as noted above, and since their parsing strategies are dependent crucially upon the use of phrase structure rules, they seem to have no choice but to give up their principle-basedness assumption. Below, I argue that by dwelling more thoroughly on the "rule-based" aspect, we can begin to get a glimpse of a processing model quite different from $F \& C$ 's Construal model, one that attains a transparent relation between the grammar and the parser.

\subsection{Access to Phrase Structure Rules}

Henceforth, let us assume that the parser operates on the basis of a "rule-based" grammar, i.e., that the parser consistently uses a set of phrase structure rules which itself constitutes the mental grammar.' There need no longer be a process of precompilation, and the parser now exhibits a transparent relationship with the grammar. As argued above, this assumption seems much more faithful to the essence of F\&C's garden path model than the poorly-grounded principle-basedness assumption.

Strict and consistent adherence to this rule-based operational characteristic of the parser, however, runs counter to an important thesis of F\&C's Construal Hypothesis, namely, that nonprimary phrases are irrelevant to structural parsing principles. In a rule-based grammar, well-formedness conditions are assumed to be represented in the form of phrase structure rules, and the rules thus represented contain both primary and nonprimary phrases. If we consistently maintain the First Analysis account of the structural parsing principles, as stated in the above quote, and continue to assume that the parser accesses all the rules concurrently and chooses the first one to become available, then it will be impossible to stick to such a thesis. If nevertheless $\mathrm{F} \& \mathrm{C}$ were to hang on, they would be obliged to conclude that nonprimary phrases

1 One should not look upon "rule-based grammars" as less explanatory than "principle-based grammars" for no sound reason. See, e.g., Gazdar et al. (1985), Crain and Fodor (1985), and Fodor and Crain (1987, 1990). 
are not "precompiled" into phrase structure rules and hence inaccessible to the parser. But apart from leaving the selective nature of the precompilation process uncertain and unaccounted for, such a conclusion is plainly false given that nonprimary phrases must be attached eventually; the parser must be able to have access to rules containing them, for, otherwise, no determinate structural analysis for them would ever be guaranteed. In point of fact, $\mathrm{F} \& \mathrm{C}$ presuppose the parser's ability to access a rule, like $\mathrm{S} \rightarrow \mathrm{Adv} \mathrm{S}$ (or IP $\rightarrow \mathrm{Adv}$ IP), which contains a nonprimary phrase when they say that "in a CP lacking an overt complementizer or inflection, an IP adverb may well lead the parser to postulate the IP node." (p. 25) Similarly, the well-known account of the garden path effect in sentences such as Without her contribution would be very inadequate presupposes the accessibility of the phrase structure rule $\mathrm{S} \rightarrow \mathrm{PP} \mathrm{S}$, which clearly contains a nonprimary phrase. It therefore seems that nonprimary phrases, as well as primary phrases, are stored in a "rule library" (Fodor and Frazier (1980)), which serves as the data structure for the parser. If so, the restriction on the applicability of structural parsing principles will hardly be tenable; it not only complicates the rule-based operation of the parser but also jeopardizes the straightforward explanation for the structural parsing principles based on the First Analysis Constraint. Hence, an obvious conclusion to draw is that the parser has access to all phrase structure rules that contain both primary and nonprimary phrases, and that the effects of the structural parsing principles, as long as they follow from the parser's parallel rule access and choice of the first available analysis, should in principle be observable with respect to both phrase types. In the following, I will pursue some consequences of this conclusion and attempt to give an outline of a possible alternative theory.

\subsection{Universality and Generality of Late Closure}

If the human parser operates uniformly in a consistently rule-based manner, i.e. analyzes incoming material by referring to phrase structure rules, then it can no longer discriminate between primary and nonprimary phrases and the structural parsing principles should apply generally, irrespective of phrase types. It is necessary, then, to account for why they appear to be inoperative in certain constructions or in certain languages. Logically, there seem to be a number of possibilities, and I will discuss some of them in what follows. But before going into the matter, we should note here that virtually all the exam- 
ples that $\mathrm{F} \& \mathrm{C}$ regard as being problematic for the structural parsing principles are in fact problematic only for Late Closure. No clear and definitive counterexample is provided for Minimal Attachment and the Minimal Chain Principle; on the contrary, Minimal Attachment is supposed to apply to nonprimary relations such as conjoined sentences and purpose clauses (see pp. 12, 42), and F\&C state that the Minimal Chain Principle applies to an extraposed relative clause, clearly a nonprimary phrase (pp. 34, 97-98). For this reason, I assume that the problem does not pertain to structural parsing principles in general, and will hereafter confine myself to Late Closure.

Thus the question now is: why is it the case that Late Closure does not appear to be operative in certain cases? One could simply abandon Late Closure and resort instead to a variety of construction-specific and language-particular strategies to accomodate the facts; but obviously, such a possibility is at once theoretically uninteresting and practically unrealistic. Taking account of the range of facts for which Late Closure can provide a simple and straightforward explanation, it will do more harm than good to give it up. Another possibility is to hypothesize that Late Closure is indeed universal but that its applicability is restricted in a certain way. F\&C's Construal Hypothesis is an instantiation of this, and according to it, Late Closure is applied to primary phrases but not to nonprimary phrases. But as noted above, such a distinction between phrase types seems extremely difficult to maintain in a strictly rule-based processing theory where the parser has immediate access to a set of phrase structure rules containing both primary and nonprimary phrases. Even if the distinction were to be maintained somehow (e.g. by storing the rules introducing primary phrases and those introducing nonprimary phrases separately in the rule library), it would hardly be possible to expect the parser to be able to sort input items coming in rapid succession into these two types instantaneously, using detailed lexical and semantic information, before making its processing decisions, i.e. before deciding whether or not to apply Late Closure. But more importantly, such a hypothesis is undesirable if Late Closure is to be derived from the First Analysis Constraint, which itself is motivated by general properties of human short-term memory. Denying the full generality of Late Closure will be tantamount to denying the generality of the mechanisms of human memory.

It is preferable, then, to be able to account for problematic cases keeping (the applicability of) Late Closure as it is. The simplest way 
will be to recognize a factor (or factors) that can counteract it; on this view, even if Late Closure applies fully generally, its effects can be immediately obscured or wiped out without obvious and detectable consequences. Imagine that a principle which exerts pressure for high attachment applies in parallel with Late Closure. Assuming that the strength of the pressure can vary in a certain way, we can cope with the parser's different preferences without modifying Late Closure itself. When the pressure is too weak (or does not reach a certain threshold), its effects cannot be observed and Late Closure prevails; but when the pressure is great enough, it overrides Late Closure and high attachment preferences will result. Thus, by postulating a high attachment preference principle (with graded strength), we can get rid of the undesirable need to restrict the applicability of Late Closure, thereby maintaining the account of it in terms of demands on short-term memory. What can be the source of high attachment preferences is not easy to pin down, and here I will only mention a few possibilities. Something like Gibson et al.'s (1996) "Predicate Proximity" principle will be a likely candidate, and if properly parameterized, it seems to help explain why relative clauses following complex heads sometimes show high attachment preferences (cf.(2)) ; the presence of intonation breaks (or commas) as well as other prosodic information serves to facilitate high attachment (cf. Marcus and Hindle (1990), Schafer et al. (1996)); and the length of the constituents to be attached seems to have some effect on the availability of high attachment readings (see Frazier and Fodor (1978: 304-305)). Notice that, interestingly, Minimal Attachment can also be regarded as providing a source of high attachment preference in examples such as Joe bought the book for Susan (Frazier and Fodor (1978), Fodor and Frazier (1980)).

The assumption here that the effects of Late Closure can be overridden by various anti-Late Closure factors implies that revising the low attachment analyses preferred by Late Closure is not necessarily costly for the parser. This is not so unlikely as it might first appear. Notice that, in the vast majority of cases, revisions of Late Closure analyses, unlike revisions of Minimal Attachment analyses, do not require the accessing and application of new, different phrase structure rules, and hence do not force the parser to postulate a host of nodes at the time of revision. For example, when the parser is forced to revise the Late Closure analysis of a sentence final adverb (as in Tom said that Bill will leave yesterday), it need not seek new rules; it can continue to use the 
current rule, something like $\mathrm{VP} \rightarrow \mathrm{VP}$ Adv, and it has only to apply the same rule, with a shift of attention, to a different VP, which will be higher in the tree structure than the original (incorrect) one. In the case of Minimal Attachment revisions, on the other hand, the parser must by definition add extra structure. If, for instance, the initial Minimal Attachment analysis of a postverbal NP as the direct object of the verb turns out to be wrong and the NP must instead be analyzed as the subject of a complement clause, then the parser must at that point have access to a set of rules involving $\mathrm{VP} \rightarrow \mathrm{V} \mathrm{S}^{\prime}, \mathrm{S}^{\prime} \rightarrow(\mathrm{Comp}) \mathrm{S}$, and $\mathrm{S}$ $\rightarrow \mathrm{NP} \mathrm{VP}$, abandoning the original rule $\mathrm{VP} \rightarrow \mathrm{V}$ NP. The difference in the mode of revision in the two cases is obvious, and it will not be very unreasonable to expect then that the difference should be reflected in the cost associated with each revision. Hence, assuming that the cost of a Late Closure revision is not considerably high in itself, we seem to be justified in claiming that a number of (as yet unspecified) factors can immediately blot out the effects of Late Closure without observable consequences. Of course it must be explained what then causes the easily observable difficulty of revising Late Closure analyses in many cases, and I will discuss this issue in detail in $\$ 3.5$.

\subsection{Association and the Current Thematic Processing Domain}

One important point about F\&C's Construal model is that it allows a limited form of delay in parsing, so that decisions about nonprimary phrases can be postponed until sufficient information becomes available. The mechanism that guarantees such a delay of decision is the operation of association into the current thematic processing domain. By this operation, the parser can temporarily wait to decide on one of a number of possible attachment sites.

But obviously, association cannot be admitted in a rule-based parser. Since it is an operation that leaves dominance and sisterhood relations unspecified, it is inherently incompatible with the use of phrase structure rules, in which dominance and sisterhood relations are fully specified. In other words, association cannot be admitted because it flouts the principle of Full Attachment (Inoue and Fodor (1995: 27)), which a rule-based parser abides by. The introduction of an operation like association will do nothing but complicate the workings of the parser; from the point of view of the uniformity and parsimony of parsing operations, it is clearly undesirable to permit the parser to perform an operation completely different from attachment. Although F\&C (pp. 
47-48) claim that the association operation is motivated by the need, arising from the limited capacity for unstructured items in short-term memory, to quickly relate an item to already analyzed material, it is not certain whether and to what extent insufficient structuring of an item by such an indeterminate operation can contribute to successful retention of the item in memory.

There are also problems with the current thematic processing domain. First, although the definition of the domain relies on the concept of theta assigners, such a concept will have to be captured by detailed lexical and/or semantic properties of input words, and the use of such (content-based) information in the first-pass parse is clearly inconsistent with a rule-based (or form-driven) parser. Note that in the garden path model as described by Rayner et al. (1983) and Frazier (1987), thematic information is used only to guide the second-pass parse (reanalysis) by a "thematic processor." Second, it is often difficult to demarcate one thematic processing domain from another due to auxiliary provisos concerning domain extension. For example, $\mathrm{F} \& \mathrm{C}$ (Ch. 4) assume that the relative clause in (11) is associated to the picture of the house, which defines the current thematic processing domain if picture is taken to be the last theta assigner:

(11) The collector lost [the picture of the house] that was so dark.

But this domain can grow larger to include the subject and the predicate, because a domain is allowed to extend if the last theta assigner is also a theta assignee ("dependent thematic domain"; pp. 165-166). Since picture is assigned a theta role by lost, the thematic processing domain now relevant to the association of the relative clause is the matrix IP, the extended projection of lost. F\&C should then predict that the subject will be almost as easily available to the relative clause as the other two NPs, for they are all in the same enlarged domain. Such a prediction will be false. (The Minimal Chain Principle can prevent the subject-host interpretation, but having recourse to it here should be impossible in the Construal model.) A similar problem arises with respect to "extended syntactic domains." F\&C (p. 147) stipulate that a lower thematic processing domain is included in the higher one provided that the specifier position of the lower domain is not filled and the syntactic category features of the two domains are compatible. According to this stipulation, the embedded CP (the extended maximal projection of the last theta assigner fixed) in (12) is included in the ma- 
trix CP (the maximal projection of the higher theta assigner say):

(12) [How did Max say [Zelda fixed the car]]?

This extended syntactic domain formation permits the extracted adjunct how to be interpreted as modifying fixed. But once the possibility of such domain extension is accepted, it becomes impossible to account for the low attachment preference as observed in (13):

(13) [Jill claimed [Jack fell yesterday]].

F\&C (pp. 56, 154) attribute the strong preference to analyze yesterday within the lower clause ("virtual late closure") to the assumption that the maximal projection of fell defines the current thematic processing domain into which association takes place. But the domain could be extended to the higher clause if an extended syntactic domain were formed as in (12), and hence yesterday would be predicted to be able to modify claimed as easily as fell, obviously a false prediction. Hence, the current thematic processing domain often does not seem to be clearly definable in cases where domain extension is involved, and by virtue of this fact, many unlikely attachment sites can easily be made available to the parser.

A third problem is concerned with the assumption that the current thematic processing domain serves as the only context within which the parser makes its decisions. F\&C (pp. 166-167) limit the context available to the parser in this way in order to account for what they call the "Sausage Machine Effects" (i.e. "Local Association" in Frazier and Fodor's (1978) and Fodor and Frazier's (1980) term). On this assumption, the parser cannot see the material outside the current domain. So in (14), for example, the parser analyzes in the library as an adjunct (nonprimary phrase) of reading, not as a locative argument (primary phrase) of put:

(14) Though Susan put the book [that John was reading] in the library ...

This is attributed to the claim that put, lying outside the current thematic processing domain defined by the bracketed relative clause, is not visible to the parser at this point. Although it may be true that the context available to the parser is limited somehow (e.g. owing to decay or forgetting of some earlier portion of the sentence), restricting the parser's sight in terms of thematic domains brings about undesirable consequences. As an example, consider (15):

(15) The professor that the student believed was arrested by the police died.

(Jackendoff and Culicover (1971: 407)) 
According to $\mathrm{F} \& \mathrm{C}$, when the parser encounters was, its sight is limited to the relative clause that the student believed, the extended projection of the last theta assigner believed. The parser would then attempt to analyze it as a primary phrase within the relative, and might initially adopt an analysis in which was is taken to be the predicate of the complement of believed (with a missing subject). If so the parser would not misanalyze was as the main verb and hence, as long as the subsequent analysis succeeds, is predicted to have little difficulty with this sentence; but such a prediction is false. Conversely, a similar parsing process would lead to the prediction that a sentence like The girl whom the boy knew left should be difficult to interpret, again a false prediction. It seems therefore that the current thematic processing domain cannot serve the purpose of defining the context available to the parser, which fact throws doubt on the usefulness of such a concept.

In view of these problems with "association" and "the current thematic processing domain," we seem to have to abandon them. But then, how can we cope with the sorts of phenomena that are supposed to be accounted for by means of association? Recall that association is an operation which, for instance, allows the parser to temporarily consider either of the NPs as the possible host for the relative clause in a sequence like NP-of-NP-Relative; since they are in the same domain, the parser can choose either as the ultimate host without difficulty. Can the parser behave in such a way without recourse to association? Below, I hope to show that it is still possible to handle the facts of this sort in a rule-based parser abiding by the principle of Full Attachment and the First Analysis Constraint, provided that the parser is equipped with a certain reanalysis mechanism incorporating some element of parallel processing.

\subsection{Reanalysis and Garden Paths}

F\&C (p. 15) note that serial models of sentence processing, including their own garden path model, must be furnished with a reanalysis mechanism to account for the fact that people eventually succeed in arriving at the correct analysis of a sentence even when it is not the initially preferred one. Since a rule-based parser obeying the First Analysis Constraint pursues a single analysis at a time, i.e. operates serially, some mechanism for reanalysis is indispensable. And it seems that consideration of such a mechanism makes it possible to have a different view of the facts that led F\&C to develop the Construal 
Hypothesis.

The logic behind F\&C's argument for the development of the Construal Hypothesis, especially of the notion "association," goes as follows: Late Closure prefers a low attachment analysis of input material; if the preferred structural analysis is revised, evidence of garden pathing should be observable; nonetheless we cannot observe clear garden path effects with respect to a certain class of phrases (nonprimary phrases); therefore, it must be concluded that Late Closure did not apply to them, and that they must have been analyzed by a completely different parsing process (i.e. association). The validity of this chain of reasoning depends on the truth of each of the premises involved, and if any of the premises is shown to be false, the argument collapses and the conclusion ceases to be tenable. As a matter of fact, we can readily question the correctness of the second premise, namely, that revising a Late Closure analysis necessarily implies the occurrence of a garden path effect. This is suspect for at least two reasons. For one thing, as we noted in $\$ 3.3$, it is likely that revisions of attachment analyses preferred by Late Closure may not be inherently costly. If this is the case, then even if the parser initially adopts a low attachment analysis following Late Closure, its revision, especially when performed quickly, may not always lead to observable difficulty. (Cf. F\&C, p. 18: "When reanalysis begins very quickly after an initial analysis is made and proceeds smoothly, the experimental techniques now available may not be able to resolve any small effect of an initial commitment to an incorrect analysis.") Hence, it will be plainly false to predict a garden path effect solely on the basis of the presence of revision. For another, there are a variety of factors, other than structural revision, that can be considered to bear on the possibility of garden pathing. Possible factors seem to include the following: the extent to which the initial analysis has been semantically interpreted by the time reanalysis is initiated (Frazier and Rayner (1982), Ferreira and Henderson (1991), Crocker (1996)), the strength of the confidence the parser has in the initial analysis (Inoue and Fodor (1995)), and the explicitness and nature of the error signal (Frazier and Rayner (1982), Fodor and Inoue (1994)). When these (and other) factors are considered, it is obviously incorrect to seek a direct link between revisions of Late Closure analyses and the occurrence of garden paths, and hence F\&C's argument for the construal model, which appears to be based on the presupposition of such a link, is also incorrect. 
How, then, can we predict when garden paths will or will not occur? And what kind of reanalysis mechanism should the parser have? Here, let us assume, basically following Inoue and Fodor (1995), that although the parser always decides immediately on a single (first) analysis, obeying the First Analysis Constraint, it can record the existence of (undeveloped) alternative analyses at each choice point, and that each analysis is assigned a value indicating the parser's confidence in it, so that the alternative options are properly ranked (or rated; see Inoue and Fodor (1995), especially pp. 33-34). On these assumptions, when the parser is forced to revise the initial analysis, it returns to a choice point and, from among the alternative analyses stored there, selects the second highest ranked one. It thus re-starts parsing, using the same mechanisms as in the first-pass parse. If this is what the parser does in case of reanalysis, then the presence or absence of a garden path can be predicted as follows. On the one hand, if the initially preferred analysis is much higher valued than the alternative, its revision is predicted to readily induce a garden path; for in such a case, the parser will have much confidence in the initial analysis and be strongly committed to it, and hence it will be very reluctant to part with the analysis. Even if it sets about reanalyzing, the alternative, being considerably lower valued, will not be easy to spot. This will be the case in examples like We remembered that the assignment will be due yesterday, where a detectable garden path is observed. The initial, low attachment analysis (following Late Closure) is more quickly and easily available to the parser than the alternative, high attachment analysis (because no anti-Late Closure force is operative here). On the assumption that the availability of an analysis is reflected in the value assigned to it, we may well expect a considerable difference in value between the two analyses, and can thus predict a garden path. Notice also that in such an example, what signals an error is not a structural ill-formedness but a pragmatic incongruity. It is suggested in Fodor and Inoue (1994) that such pragmatic cues are not of great help to the parser in case of reanalysis. On the other hand, we can predict that the parser will fail to exhibit clear garden path effects in cases where the value assigned to the initial analysis is not very high and hence the parser's confidence in it is low; for then the selection of the second alternative, which may be almost as highly valued as the initial analysis, will be performed quickly and smoothly, without any sign of difficulty. For example, when the parser encounters a relative clause after a sequence 
$\mathrm{NP}_{1}-\mathrm{P}-\mathrm{NP}_{2}$, it will initially adopt the analysis where $\mathrm{NP}_{2}$ is taken to be the host for the relative clause, but at the same time record an alternative analysis in which $\mathrm{NP}_{1}$ is regarded as the host; if later material turns out to be inconsistent with the initial analysis, the parser switches to the alternative. Assuming that the value assigned to the initial (low attachment) analysis is not very high, or does not differ much from that assigned to the alternative analysis (perhaps owing to a principle like Predicate Proximity), we expect that this revision will be next to costfree: there will be no clearly observable garden path. The parser obeyed Late Closure and chose a single (first) analysis and yet it appears as if it hadn't; it behaves as if it were able to consider multiple alternative analyses at a time. But such a behavior results simply from the manner in which reanalysis is executed and not from a novel component that can perform a non-rule-based operation like association.

I suspect that much of the evidence that F\&C attribute to construal/ association will be interpretable along these lines. If so, apparent instances of attachment indeterminacy, as observed with relative clauses following complex heads, extraposed relative clauses, secondary predicates, and so on, can no longer serve as the unequivocal grounds on which to claim that the parser must include a special gadget for dealing with them; for they can now be interpreted as reflecting the rapid and efficient manner in which the parser recovers from its initial misanalysis. Certainly, there remain a number of unclear points at present and we need further elaboration (e.g., Exactly what are the determinants of the value assigned to each of the alternative analyses? And how do they interact when they conspire to rank the alternatives appropriately?), but what is important is that a thorough investigation of the reanalysis mechanism will save our rule-based parser from being modified and complicated unnecessarily. If the parser performs reanalysis as described above, it operates on the second pass in much the same way as it does on the first. There is no need to require the parser to make different processing decisions according to the types of phrases it encounters, and hence the theory need not have two distinct decisionmaking devices. We can continue to claim that the parser operates uniformly in a rule-based manner and consistently makes its decisions (with variable confidence) following the First Analysis Constraint and the principle of Full Attachment, and the result is a parsing model much more parsimonious than F\&C's. 


\subsection{Interpretive Principles}

In the Construal model, a nonprimary phrase associated into the current thematic processing domain is processed using a variety of interpretive principles, and thus its (initial) analysis is guided and influenced by semantic and discourse-pragmatic information (referentiality, discourse salience, focus, plausibility, etc.; see \$2.2). Although it may be easy to find fault with the particulars of the interpretation processes and principles that $\mathrm{F} \& \mathrm{C}$ actually propose, no theory of sentence comprehension can deny that such information must somehow be made available to the parser (for otherwise no coherent and complete interpretation might be guaranteed). So, rather than being too critical, I would simply appreciate $\mathrm{F} \& \mathrm{C}$ 's attempt to inquire into the complexities of interpretation processes and to formulate concrete principles to account for them. But now that association is thrown away, we must take a different look at the way such principles are brought to bear on parsing.

In the model I have been describing, the parser makes its initial attachment decisions (concerning both primary and nonprimary phrases) following the First Analysis Constraint, i.e. based exclusively on structural information. Therefore, we cannot say that semantic-pragmatic information guides the initial analysis. An obvious alternative way to make such information available to the parser will be to have interpretive principles take part, on the second pass, in the evaluation of the alternative analyses that the parser has recognized; by permitting such principles to influence the selection that the (second-pass) parser makes from among the non-initial analyses (which have been stored at a choice point), we can expect them to function as reanalysis-facilitators. Imagine that, for example, the parser is analyzing an $\mathrm{NP}_{1}-\mathrm{P}-\mathrm{NP}_{2}-\mathrm{Rela}-$ tive sequence, which can be ambiguous as to which NP is the antecedent of the relative clause. The analysis that the parser initially adopts is the one in which $\mathrm{NP}_{2}$ is taken to be the antecedent, but along with it, the parser records the existence of an alternative analysis where $\mathrm{NP}_{1}$ is to be the antecedent. When this alternative analysis is in accordance with some interpretive principle (cf. (7)), the second-pass parser will be encouraged to reanalyze and unhesitatingly shift to it if the two analyses have been almost equally highly valued by the first-pass parser. (Even if the initial analysis has been more highly valued in the first-pass parse, interpretive success may give the parser some incentive to reanalyze.) Thus, in a parser with a capacity to store multiple alterna- 
tive analyses, we can utilize interpretive principles to facilitate the parser's attempt at reanalysis by having them pinpoint which of the alternative analyses best fits in with the semantic-pragmatic requirements, especially when the alternatives do not differ greatly in terms of the first-pass parser's evaluation based on formal/structural measures. It goes without saying that this hypothesis is only a speculation, and more work needs to be carried out. But as long as the parser makes its initial decisions following the First Analysis Constraint and Full Attachment, such a way of looking at interpretive principles will be unavoidable.

\section{Summary and Conclusion}

Construal is a book containing a wide range of new data and insights that are well worthy of close scrutiny, and the hypothesis $\mathrm{F} \& \mathrm{C}$ propose in it is sure to stimulate later work in psycholinguistics and to help broaden the range of construction types that must be addressed by theories of the human sentence processing mechanism. Especially, their approach to the processing of adjuncts, identified as nonprimary phrases, will draw attention to a number of phrases and constructions which have so far lain outside psycholinguists' sight and to the identification of relevant semantic and pragmatic factors. At least in this respect, $\mathrm{F} \& \mathrm{C}$ 's contribution cannot be emphasized too much.

However, the particular approach that they take seems to be undesirable, mainly from a conceptual point of view, in that it forces one to give up the generality of parsing principles and the uniformity of the parser's syntactic operations: F\&C's Construal Hypothesis deprives Late Closure of generality by narrowing its applicability, and destroys the uniformity of the parser's operation by allowing it to perform a syntactic operation fundamentally distinct from attachment. Such a hypothesis is to be avoided not only because it leads to a complicated and unparsimonious theory but also because it is inconsistent with the assumption that the parser operates in a rule-based manner (essentially as in their garden path model). I have advocated an alternative model that is expected to cope with most of the facts $F \& C$ address without undesirable additions and modifications to the rule-based operational characteristic of the parser. It has been proposed that the model should have the following two properties. First, it must incorporate several anti-Late Closure principles which apply in parallel with Late 
Closure and sometimes override it. The postulation of such principles will render it unnecessary to restrict the applicability of Late Closure, thereby maintaining its full generality. Second, the model must be equipped with a quick and efficient reanalysis mechanism, in particular, the ability to record and evaluate multiple, if not all, alternative analyses (which are not necessarily fully developed). Provided that the parser can recover from its initial misanalysis quickly and smoothly without detectable difficulty, we can dispense with a special syntactic operation like association and go on claiming that the parser operates uniformly, i.e. performs attachment analyses regardless of the types of phrases it encounters. Many things remain to be done to be sure, but the model advocated here will serve as a basis for further attempts to seek a conceptually favorable theory of the human sentence processing mechanism.

\section{REFERENCES}

Berwick, Robert C. and Amy S. Weinberg (1984) The Grammatical Basis of Linguistic Performance: Language Use and Acquisition, MIT Press, Cambridge, MA.

Crain, Stephen and Janet Dean Fodor (1985) "How Can Grammars Help Parsers?" Natural Language Parsing: Psychological, Computational, and Theoretical Perspectives, ed. by David R. Dowty, Lauri Karttunen and Arnold M. Zwicky, 94-128, Cambridge University Press, Cambridge.

Crocker, Matthew, W. (1996) Computational Psycholinguistics: An Interdisciplinary Approach to the Study of Language, Kluwer Academic Publishers, Dordrecht.

Cuetos, Fernando and Don C. Mitchell (1988) "Cross-Linguistic Differences in Parsing: Restrictions on the Use of the Late Closure Strategy in Spanish," Cognition 30, 73-105.

Ferreira, Fernanda and John M. Henderson (1991) "Recovery from Misanalyses of Garden-Path Sentences," Journal of Memory and Language 30, 725-745.

Fodor, Janet Dean (1989) "Empty Categories in Sentence Processing," Language and Cognitive Processes 4, 155-209.

Fodor, Janet Dean and Stephen Crain (1987) "Simplicity and Generality of Rules in Language Acquisition," Mechanisms of Language Acquisition, ed. by Brian MacWhinney, 35-63, Lawrence Erlbaum Associates, Hillsdale, NJ.

Fodor, Janet Dean and Stephen Crain (1990) "Phrase Structure Parameters," Linguistics and Philosophy 13, 619-659. 
Fodor, Janet Dean and Lyn Frazier (1980) "Is the Human Sentence Parsing Mechanism an ATN?" Cognition 8, 417-459.

Fodor, Janet Dean and Atsu Inoue (1994) "The Diagnosis and Cure of Garden Paths," Journal of Psycholinguistic Research 23, 407-434.

Frazier, Lyn (1987) "Sentence Processing: A Tutorial Review," Attention and Performance XII: The Psychology of Reading; ed. by Max Coltheart, 559-586, Lawrence Erlbaum Associates, Hillsdale, NJ.

Frazier, Lyn (1989) “Against Lexical Generation of Syntax," Lexical Representation and Process, ed. by William Marslen-Wilson, 505-528, MIT Press, Cambridge, MA.

Frazier, Lyn and Janet Dean Fodor (1978) "The Sausage Machine: A New Two-Stage Parsing Model," Cognition 6, 291-325.

Frazier, Lyn and Keith Rayner (1982) "Making and Correcting Errors during Sentence Comprehension: Eye Movements in the Analysis of Structurally Ambiguous Sentences," Cognitive Psychology 14, 178-210.

Frazier, Lyn and Keith Rayner (1988) "Parameterizing the Language Processing System: Left- vs. Right-Branching within and across Languages," $E x$ plaining Language Universals, ed. by John A. Hawkins, 247-279, Blackwell, Oxford.

Gazdar, Gerald, Ewan Klein, Geoffrey K. Pullum, and Ivan A. Sag (1985) Generalized Phrase Structure Grammar, Basil Blackwell, Oxford.

Gibson, Edward, Neal Pearlmutter, Enriqueta Canseco-Gonzalez, and Gregory Hickok (1996) "Recency Preference in the Human Sentence Processing Mechanism," Cognition 59, 23-59.

Grimshaw, Jane (1993) "Minimal Projection, Heads, and Optimality," ms., Rutgers University.

Inoue, Atsu and Janet Dean Fodor (1995) "Information-Paced Parsing of Japanese," Japanese Sentence Processing, ed. by Reiko Mazuka and Noriko Nagai, 9-63, Lawrence Erlbaum Associates, Hillsdale, NJ.

Jackendoff, Ray S. and Peter Culicover (1971) "A Reconsideration of Dative Movements," Foundations of Language 7, 397-412.

Marcus, Mitchell and Donald Hindle (1990) "Description Theory and Intonation Boundaries," Cognitive Models of Speech Processing, ed. by Gerry T. M. Altmann, 483-512, MIT Press, Cambridge, MA.

Rayner, Keith, Marcia Carlson, and Lyn Frazier (1983) "The Interaction of Syntax and Semantics during Sentence Processing: Eye Movements in the Analysis of Semantically Biased Sentences," Journal of Verbal Learning and Verbal Behavior 22, 358-374.

Schafer, Amy, Juli Carter, Charles Clifton Jr., and Lyn Frazier (1996) "Focus in Relative Clause Construal," Language and Cognitive Processes 11, $135-163$. 
Department of English

Tokyo Metropolitan University

Minami Osawa, Hachioji-shi

Tokyo 192-0397 